\title{
GPR143 mutations in Chinese patients with ocular albinism type 1
}

\author{
XIUHUA JIA $^{1 *}$, JIN YUAN $^{2 *}$, XIAOYUN JIA ${ }^{2}$, SHIQI LING ${ }^{1}$, SHIQIANG LI $^{2}$ and XIANGMING GUO ${ }^{2}$ \\ ${ }^{1}$ Department of Ophthalmology, The Third Affiliated Hospital, Sun Yat-sen University, Guangzhou, Guangdong 510630; \\ ${ }^{2}$ State Key Laboratory of Ophthalmology, Zhongshan Ophthalmic Center, \\ Sun Yat-sen University, Guangzhou, Guangdong 510060, P.R. China
}

Received January 25, 2016; Accepted February 3, 2017

DOI: $10.3892 / \mathrm{mmr} .2017 .6366$

\begin{abstract}
The aim of the present study was to evaluate mutations of the G protein-coupled receptor 143 (GPR143) gene for ocular albinism type 1 (OA1) in Chinese patients. For the current study, 8 patients with OA1 were selected from the database of ocular genetic diseases. Genomic DNA of OA1 was prepared from venous leukocytes collected from the patients. Cycle sequencing was used to analyze the exons and adjacent introns of GPR143. The variation detected was analyzed by bidirectional DNA sequencing and further evaluated in 96 controls using heteroduplex-single strand conformational polymorphism analysis. Additionally, slit lamp photography of anterior segment, fundus photography and optical coherence tomography (OCT) were performed to identify the clinical features of OA1. In five patients with OA1, 5 GPR143 gene mutations were identified and four of them there were novel mutations. The screening rate is $62.5 \%$, including c.333G $>\mathrm{A}$ (p.W111X), c.353G $>\mathrm{A}$ (p.G118E) (known mutation), C.658+2T $>\mathrm{G}$ (splice mutation), c.215_216insCGCTGC (p.71-72insAA) and c.17T >C (p. L6P). These mutations were absent in the 96 normal controls. Only one patient with OA1 in the present study was female. Patients with OA1 often have congenital nystagmus, refractive error, severe decline of visual acuity (from 0.1 to 0.4 ) and foveal hypoplasia. Different degrees of pigment loss were evident in the patients' iris and retina, whereas macular structure was not identified in the OCT examination. The findings of the present study expanded the gene mutation spectrum of GPR143 and investigated the clinical phenotype of patients with OA1 in the Chinese population. Additional evidence
\end{abstract}

Correspondence to: Professor Xiangming Guo, State Key Laboratory of Ophthalmology, Zhongshan Ophthalmic Center, Sun Yat-sen University, 54 Xian Lie Nan Road, Guangzhou, Guangdong 510060, P.R. China

E-mail: zocguoxm@aliyun.com

*Contributed equally

Key words: Ocular albinism type 1, G protein-coupled receptor 143 gene, genetic variation for clinical diagnosis was provided along with differential diagnosis and genetic counseling.

\section{Introduction}

Ocular albinism type 1 (OA1) is an X-linked disorder (1), which is the most common form of ocular albinism, with an estimated prevalence of 1 in 60,000 at birth (2). OA1 is characterized by a severe reduction in visual acuity, refractive errors, nystagmus, iris translucency, fundus hypopigmentation, foveal hypoplasia and loss of stereoscopic vision due to misrouting of the optic fibers at the optic chiasm (3-6).

In the sclera, the performance of the iris and fundus is crucial. The majority of patients have evident clinical features of pronounced pigment loss in the iris and retina, which may be distinguished from patients with congenital motor nystagmus (CMN). However, the performance of the iris and retinal depigmentation is not typical and varies greatly among patients. Additionally, congenital nystagmus is frequently clinically misdiagnosed as OA1 or CMN in China, although the genetic and clinical manifestations of these diseases differ significantly.

OA1 occurs due to mutations in the ocular albinism I G protein-coupled receptor 143 (GPR143) gene (Online Mendelian Inheritance in Man no. 300500). This $\mathrm{G}$ protein-coupled receptor protein is expressed in ocular and epidermal melanocytes and may be a melanosomal transmembrane protein $(7,8)$. GPR143 is a pigment cell-specific intracellular glycoprotein consisting of 404 amino acid residues that is mutated in patients with OA1 (9).

The aim of the present study was to identify mutations in the exons and exon/intron junctions of the OA1 gene using cycle sequencing, clinically evaluate the characteristics of the OA1 patients, including female carriers and provide additional evidence for future clinical and differential diagnoses, and genetic counseling of this disease. A total of five mutations in the GPR143 gene were identified in the present study, four of these were previously unknown mutations.

\section{Materials and methods}

Patients and clinical data. A total of 8 patients with OA1 and 96 normal controls, mainly from the south of China, participated in the present study. These individuals, recruited between August 2008 and July 2011, were 
identified by the Pediatric and Genetic Clinic of Zhongshan Ophthalmic Center (Sun Yat-sen University, Guangzhou, China). Informed consent conforming to the Declaration of Helsinki was obtained from each participant prior to the study. The medical and ophthalmic histories of the patients were obtained. A detailed ophthalmological examination, including slit lamp photography of the anterior segment, fundus photography, optical coherence tomography (OCT) and examination of visual acuity, were used to identify the clinical features of OA1.

Genetic mutation screening. Genomic DNA was prepared from leukocytes, collected from $5 \mathrm{ml}$ peripheral venous blood obtained from the patients, using the phenol-chloroform extraction method (10). The primers (Takara Bio, Inc., Otsu, Japan) for GPR143 (Table I) were used with a polymerase chain reaction (PCR) Amplification kit (Takara Bio, Inc.) to amplify the coding exons (exon 1 to exon 9) of GPR143 and adjacent intronic sequences of the gene (human genome build 36.3, NC_000023.10 for gDNA, NM_000273.2 for cDNA, NP_000264.2 for protein). The PCR reaction was performed in a thermocycler (Biometra $\mathrm{GmbH}$, Göttingen, Germany) under the following conditions: Initial denaturation at $94^{\circ} \mathrm{C}$ for $5 \mathrm{~min}$ followed by $35-37$ cycles of $94^{\circ} \mathrm{C}$ for $30 \mathrm{sec}$, primer-specific annealing temperature (Table I) for $30 \mathrm{sec}$, and $72^{\circ} \mathrm{C}$ for $30 \mathrm{sec}$. The final extension cycle was performed at $72^{\circ} \mathrm{C}$ for $5 \mathrm{~min}$. The PCR products of the exons and adjacent intronic sequences for each patient were sequenced with the ABI BigDye Terminator cycle sequencing kit v3.1 (Applied Biosystems; Thermo Fisher Scientific, Inc., Waltham, MA, USA) according to the manufacturer's protocol, using an ABI Prism 3100 sequencer and the results were manually confirmed. The sequencing results from each patient and the consensus sequences of GPR143 from the National Centre for Biotechnology Information (NCBI) human genome database (NM_000273.2) were imported into the SeqMan II software (DNAStar, Inc., Madison, WI, USA), using the Lasergene 8.0 package (DNAStar, Inc.) and were aligned to identify genetic variations. Each mutation was confirmed by bidirectional sequencing, and novel mutations were named according to the nomenclature recommended by the Human Genome Variation Society (http://www.hgvs.org/).

Determination of changes in genetic information. The sequences containing genetic variations and the standard sequences obtained from the NCBI human genome database were imported into MapDraw of the Lasergene package to identify the impact on amino acid coding. To estimate the conservation of the mutation sites using ClustalW, protein sequences from different species were identified from the NCBI website and entered into the Lasergene package MegAlign program (DNAStar, Inc.). Differences in the amino acids and the pathogenicity of each mutation were evaluated using the Blosum 62 matrix (http://www.uky. edu/Classes/BIO/520/BIO520WWW/blosum62.htm) and the PolyPhen (http://genetics.bwh.harvard.edu/pph/) analytical tool, respectively.

Heteroduplex-single strand conformational polymorphism (HA-SSCP) analysis. The genetic variations that were identified in the GPR 143 gene were evaluated in the 96 normal controls and in the patients that screened positive for genetic variations using HA-SSCP as previously described (11-13). DNA fragments of the mutated sites were PCR-amplified aforementioned. The PCR products were mixed with an equal volume of gel loading buffer $(95 \%$ formamide, $20 \mathrm{mM}$ EDTA, $0.05 \%$ bromophenol blue and $0.05 \%$ xylene cyanol), denatured at $95^{\circ} \mathrm{C}$ for $5 \mathrm{~min}$ and immediately placed on ice for $5 \mathrm{~min}$. Samples ( $3 \mu \mathrm{l} / \mathrm{well}$ ) were loaded directly onto $8 \%$ polyacrylamide gels and gel electrophoresis was conducted at $40 \mathrm{~W}$ for $8 \mathrm{~h}$, at room temperature, in a solution of $0.5 \mathrm{X}$ Tris/borate/EDTA buffer.

\section{Results}

Mutation analysis. In the present study, five patients with OA1 were determined to have genetic mutations in the GPR143 gene, with four out of the five mutations being novel. The screening rate was $62.5 \%$ for the following mutations: C.333G $>$ A (p.W111X), c.353G $>$ A (p.G118E) (known mutation), C.658+2T $>\mathrm{G}$ (splice mutation), c.215_216 insCGCTGC (p.71-72insAA) and c.17T >C (p. L6P) (Fig. 1).

The protein in this position was highly conserved based on the comparative analysis with 7 orthologs from different mammalian species (Fig. 2). HA-SSCP was also used to screen the 96 unaffected control individuals (Fig. 3); however, no mutations were identified.

PolyPhen and Blosum 62 analyses were used to determine if whether the missense mutations identified in the present study resulted in changes in the residue weight at the protein level. Blosum 62 was used to evaluate the missense mutations and PolyPhen was used to determine whether an amino acid change had a 'probable damaging' effect. It was determined that the p.G118E and p.L6P mutations may be damaging with a score 0.999 by PolyPhen and they led to a change in the residue weighting from 6 to- 2 and from 4 to- 3 by Blosum 62 , respectively.

Clinical phenotype. In the present study, all patients with OA1 exhibited different degrees of horizontal nystagmus; however, they typically manifested a severe decline in visual acuity. Uncorrected visual acuity ranged from 0.1 to 0.3 , and corrected visual acuity ranged from 0.1 to 0.4 (Table II).

Upon slit lamp examination, different degrees of abnormal pigmentation were observed in the irises of patients. Peripheral iris depigmentation, such as a ring (which was irregular in some patients) or fan, was observed, although the pigmentation of the iris around the pupil area appeared normal and the overall iris color appeared darker than normal (Fig. 4A). However, some patients did not have obvious depigmentation of the iris; therefore, determining whether the patient had OA1 or was healthy was difficult in such cases (Fig. 4B). Iris depigmentation did not lead to iris transillumination in the present study (Fig. 4).

Based on the findings of the present study, patients with OA1 have different degrees of retinal depigmentation as determined by fundus examination. Hypopigmentation of the retina was universal and the choroidal blood vessels were clearly visible, which is different from the phenotype of oculocutaneous albinism. Diffuse reduction of pigmentation 


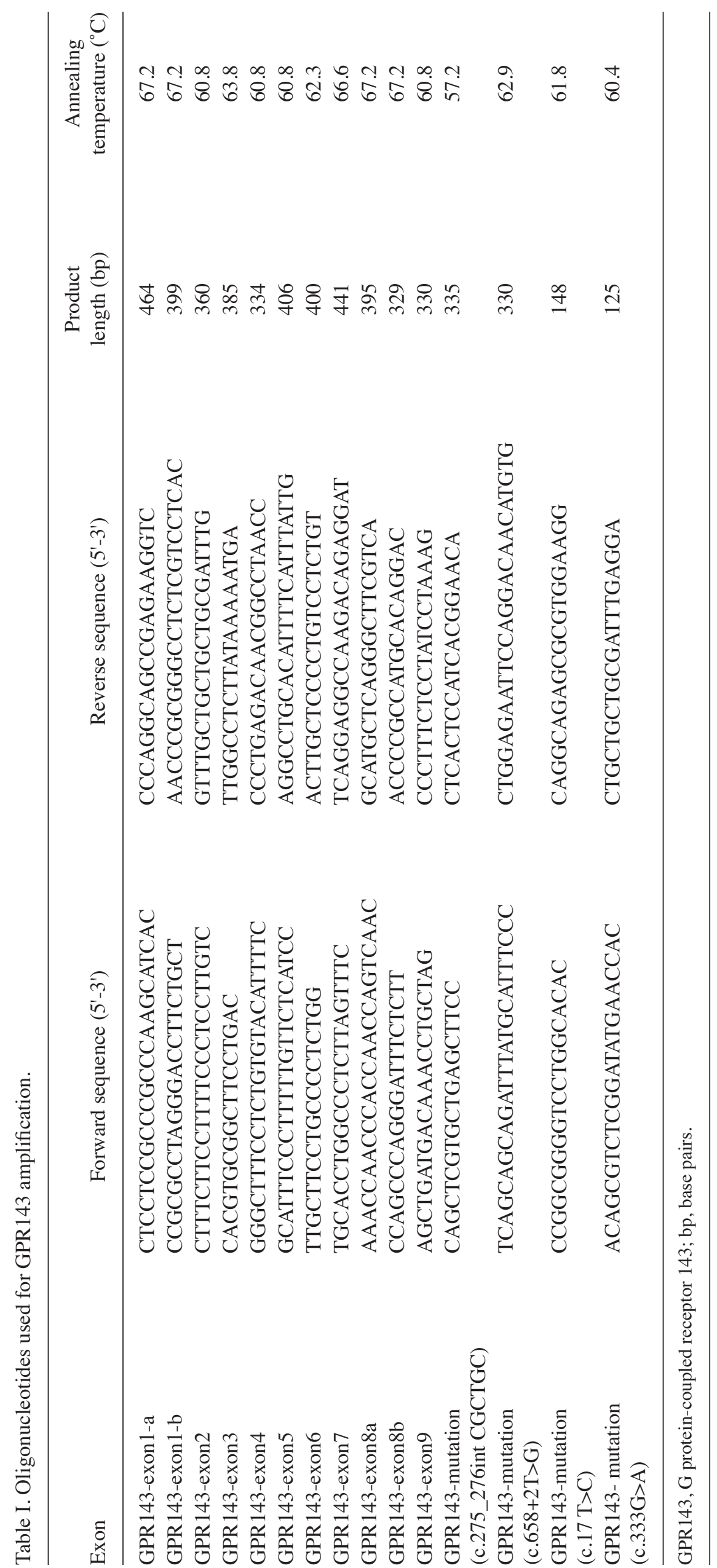


$\mathbf{A}$

Normal

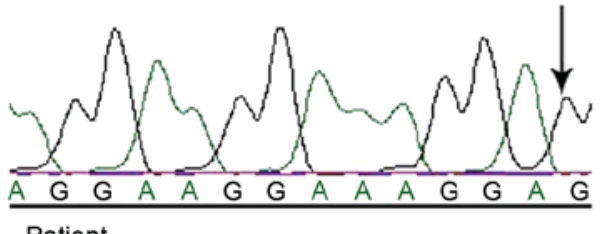

Patient

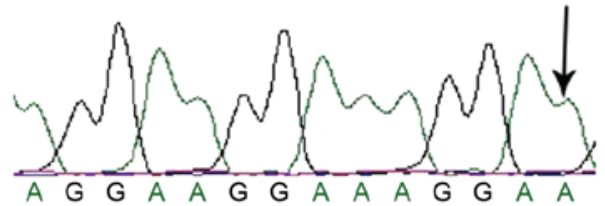

C

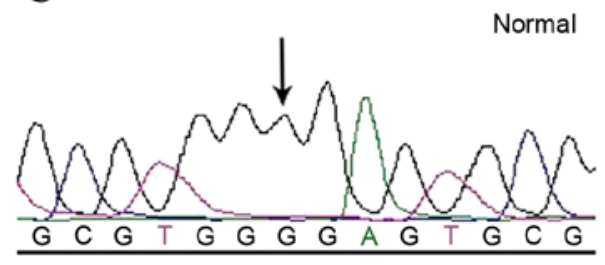

Patient

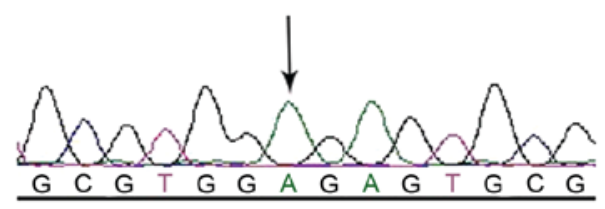

$\mathbf{E}$
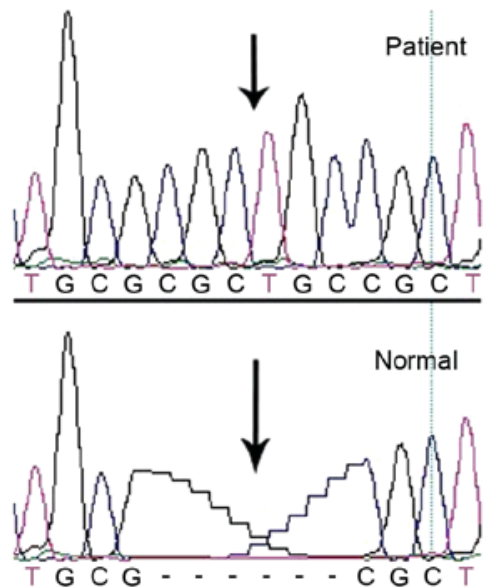

B

Patient
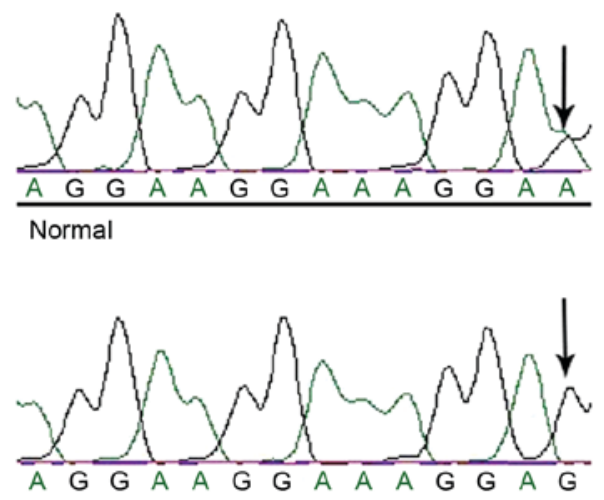

D

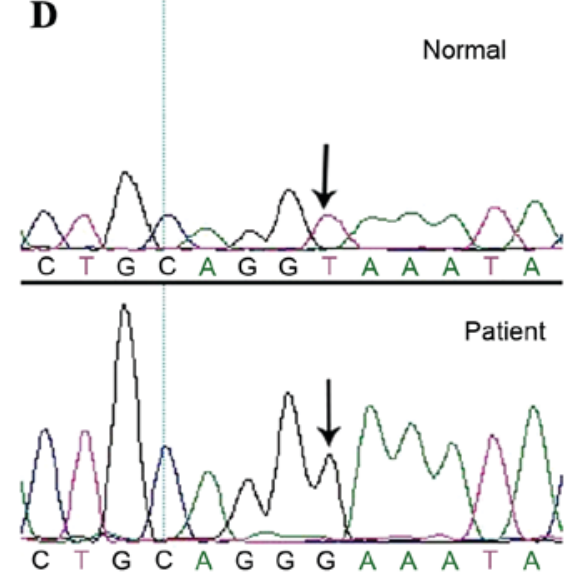

$\mathbf{F}$

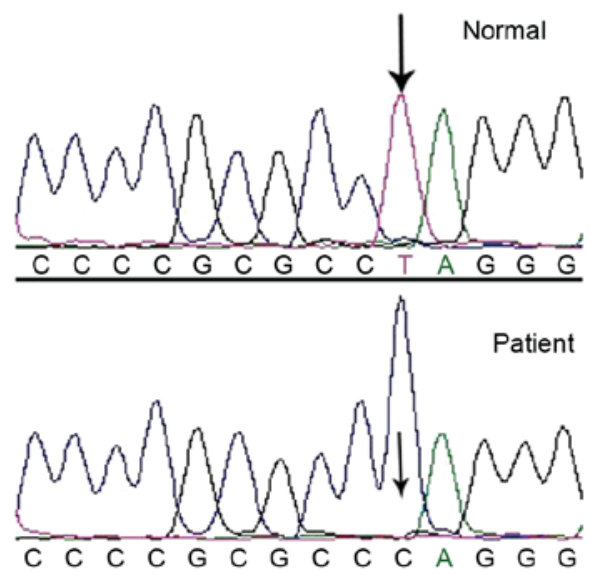

Figure 1. GPR143 cycle sequencing results. (A) Homozygous c.333G>A (p.W111X) mutation, indicated by the lower arrow, was identified in the OA1 patient and confirmed by bidirectional sequencing. The upper arrow represents the corresponding normal allele from an unaffected control individual. (B) Heterozygous c.333G $>$ A (p.W111X) mutation, indicated by the upper arrow, was identified in the OA1 patient and confirmed by bidirectional sequencing. The lower arrow represents the corresponding normal allele from an unaffected control individual. (C) c.353G>A (p.G118E) mutation was identified in the OA1 patient, which is indicated by the lower arrow, and confirmed by bidirectional sequencing. The upper arrow indicates the corresponding normal sequence from an unaffected control individual. (D) c.658+2T $>\mathrm{G}$ mutation, which is indicated by the lower arrow, was identified in the OA1 patient and confirmed by bidirectional sequencing. The upper arrow highlights the corresponding normal sequence from an unaffected control individual. (E) c.215_216 ins CGCTGC (p.71-72intAA) mutation, which is indicated by upper arrow, was identified in the OA1 patient and confirmed by bidirectional sequencing. The lower arrow represents the corresponding normal sequence from an unaffected control individual. (F) c.17T $>C$ (p. L6P) mutation, which is indicated by the lower arrow, was identified in the OA1 patient and confirmed by bidirectional sequencing. The upper arrow indicates the corresponding normal sequence from an unaffected control individual. OA1, ocular albinism type 1 . 
A

Bos taurus
Homo sapiens
Macaca mulatta
Mus musculus
Rattus norvegicus
Pongo abelii

\section{B}

Bos taurus

Homo sapiens

Macaca mulatta

Mus musculus

Rattus norvegicus

Pongo abelii
MASPRLLGTFCCPTRDAATQLA MASPRLLGTFCCPTRDAATQLV MASPRL GTFCCPTRDAATQLV MASPRL GIFCCPTWDAATQLV MASPRLL GIFCCPSWDAATQLV MASPRL GTFCCPTRDAATQLV

PSFVDDISAVNNTDVWPAVFC
VSDMNHTEIWPAAFCVGSAMW
ISDVNRTEIWPAVFCVGSAMW
ISNVNATDIWPATFCVGSAMW
ISNMNGTDIWPTAFCVGSAMW
VSDMNHTEIWPAAFCVGSAMW

Figure 2. Analysis of mutation conservation. Comparison of the genetic sequences of seven orthologs from different mammalian species. (A) p. L6P mutation involves a highly conserved amino acid in the GPR143 gene, highlighted by the box. (B) p.G118E mutation does not involve a conserved amino acid in the GPR143 gene. GPR143, G protein-coupled receptor 143.

A

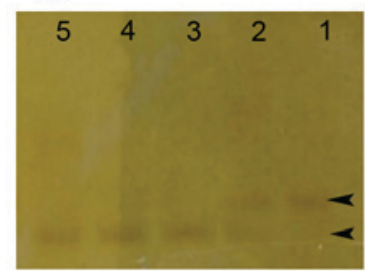

C

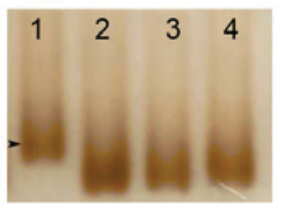

B

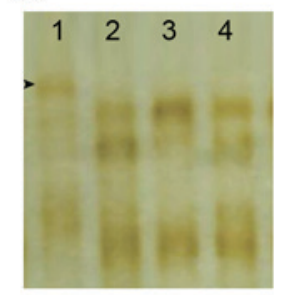

D

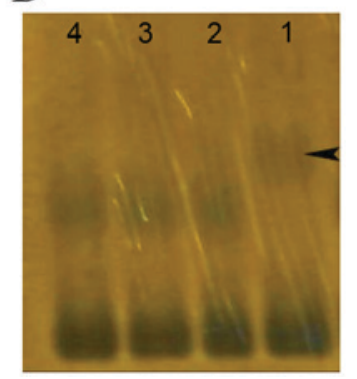

Figure 3. HA-SSCP analysis of the GPR143 genetic mutations in patients with OA1, arrows indicate the differential bands. (A) Lane 1, band from a patient; lane 2, the bands from a carrier with the c.333G $>$ A (p.W111X) mutation. Lanes 3-5, bands from normal controls. (B) Lane 1, band from a patient with the c. $658+2 \mathrm{~T}>\mathrm{G}$ mutation. Lanes $2-4$, bands from normal controls (C) HA-SSCP analysis of the GPR143 genetic mutation in a patient with OA1. Lane 1, band from a patient with the c.215_216 int CGCTGC (p.71-72intAA) mutation. Lanes 2-4, bands from normal controls. (D) Lane 1, band from a patient with the c.17T $>C$ (p. L6P) mutation. Lanes 2-4, bands from normal controls. HP-SSCP, heteroduplex-single strand conformation polymorphism; OA1, ocular albinism type 1; GPR143, G protein-coupled receptor 143 .

in the retina, but not in the choroidal vessels, was clearly observed, as the color of the retina was slightly redder than the healthy tissue. The entire retina exhibited features similar to highly myopic eyes, such as irregular retinal depigmentation and hyperpigmentation in some regions. In particular, the retina exhibited regional depigmentation around the optic
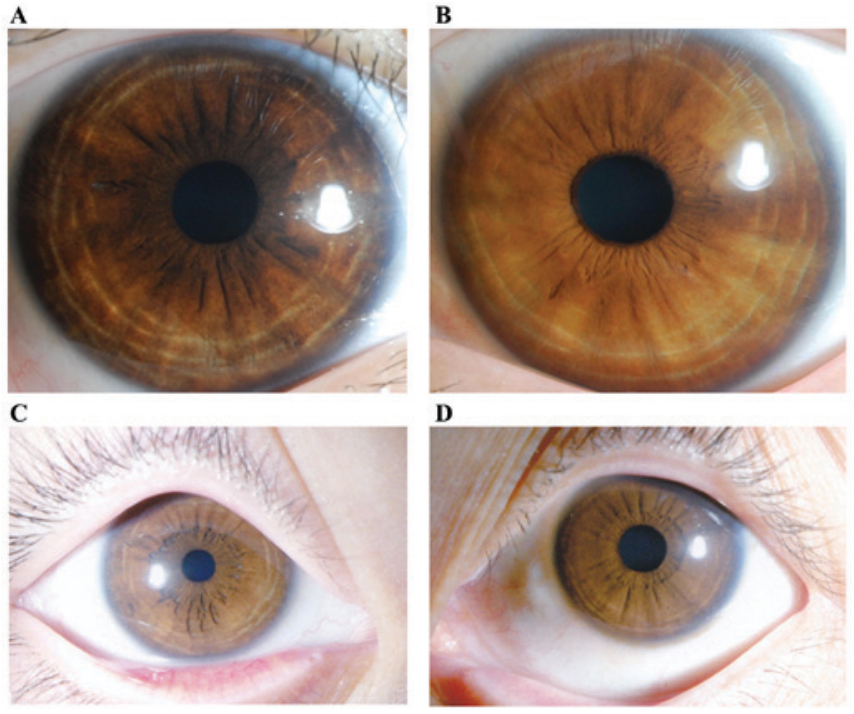

Figure 4. Iris images of patient with OA1. (A) Patient iris exhibited peripheral iris depigmentation, in the shape of a ring or fan, with pigmentation of the iris around the pupil area appearing normal and an overall iris color that appeared darker than normal. (B) Iris of the patient did not have obvious depigmentation. Therefore, determining whether this patient had OA 1 or was healthy was difficult. (C and D) Irregular ring depigmentation in the peripheral iris was observed in the patient. OA1, ocular albinism type 1.

disc and peripheral retina, where the choroidal vessels may be observed (Fig. 5). The macular foveal structure was not visible in OCT examination (Fig. 6).

\section{Discussion}

The GPR143 gene encodes a pigment cell-specific G protein-coupled receptor protein (GPCR) that is localized intracellularly in melanosomes. OA1 has been reported to regulate melanosome formation in the biogenesis stage, as well as the motility of melanosomes (14).

Genetic analysis has detected $>60$ mutations in the GPR143 gene in sporadic and familial cases. Previous studies have determined that the GPR143 mutations are deletion mutations and that most of these deletion mutations are point mutations $(1,15-17)$. In these mutants, large fragment deletion mutations were not uncommon. However, the present study identified one case of an insertion mutation. A potential explanation may be due to ethnic differences and the biased selection of the volunteers in the present study. A previous study demonstrated that the OA1 protein was a GPCR protein that spans the integral membrane seven times, with its $\mathrm{C}$ terminus oriented towards the cytoplasm, and this protein may function as an intracellular protein localized in endolysosomal organelles (18).

In the present study, the GPR143 mutations c.333G $>\mathrm{A}$ (p.W111X), c.353G>A (p.G118E) and c.17T>C (p.L6P) were located in the loop regions within the lumen of melanosomes or lysosomes. The missense mutations, p.G118E and p.L6P, were relatively highly conserved in seven different mammalian species and had high pathogenicity, as determined by Blosum 62 and PolyPhen. It is possible that the missense mutations c.353G >A (p.G118E) and c.17T>C (p.L6P) may alter genetic receptor binding, which may lead to functional 
Table II. Visual acuity of patients with OA1.

\begin{tabular}{lcccc}
\hline Patient ID & Gender & Visit age (years) & Naked vision & Corrected vision \\
\hline QT299 & Male & 6 months & Perception of light & N/A \\
QT418 & Male & 7 & $0.2 / 0.2$ & $0.2 / 0.2$ \\
QT593 & Male & 43 & $0.1 / 0.1$ & $0.1 / 0.1$ \\
QT638 & Female & 10 & NA & $0.4 / 0.4$ \\
QT646 & Male & 8 months & Perception of light & N/A \\
QT676 & Male & 2 months & N/A & $0.1 / 0.3^{-}$ \\
QT720 & Male & 10 & $0.1 / 0.1$ & $0.3^{+}$ \\
QT751 & Male & 7 & 0.2 & \\
\hline
\end{tabular}

OA1, ocular albinism type 1 .
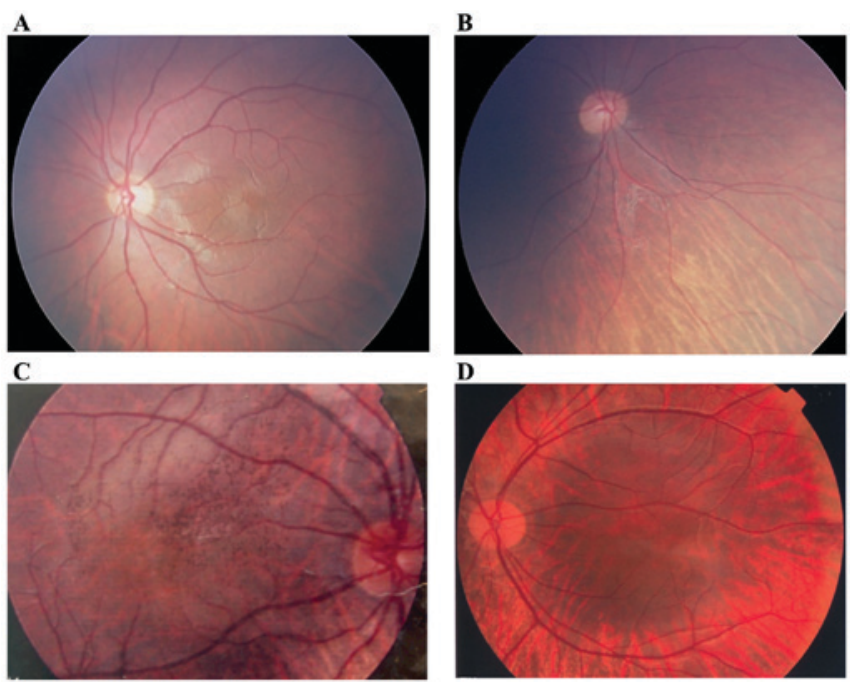

Figure 5. Fundus images from patients with OA1. (A) Diffuse reduction in the pigmentation of the retina, was clearly observed in the retina of a patient as the color of the retina was slightly redder compared with healthy tissue. (B) Retina of the patient exhibited a universal loss of retinal pigment, resulting in the choroidal blood vessels being clearly visible; however, this retina pigmentation pattern was not representative of oculocutaneous albinism based on the fundus examination. (C) Retina of the patient exhibited irregular retinal depigmentation with the entire retina having a deep red color, similar to highly myopic eyes. (D) Retina of the patient exhibited regional depigmentation, which was evident around the optic disc and periphery where the choroidal blood vessels were visible; however, the macular pigment was normal. OA1, ocular albinism type 1 .

changes; however, further investigation is required to confirm this conclusion as the predictions of the current study are based only on bioinformatics data, requiring experimental support. The insertion mutation, c.215_216 ins CGCTGC (p.71-72insAA), is located in transmembrane region (TM) 2. Systematic and experimental topology analyses may be used in future studies to identify the function of the TM domains in terms of GPCR functionality, screen ligands and identify proteins located in OA1 signaling pathways.

The GPR143 gene has been previously reported as being characterized by a high mutation rate of up to $95 \%$ (19). The present study identified a mutation rate of $62.5 \%$, which may be primarily due to the small sample size, probands from sporadic cases, or bias in the selection of volunteers. GPR143

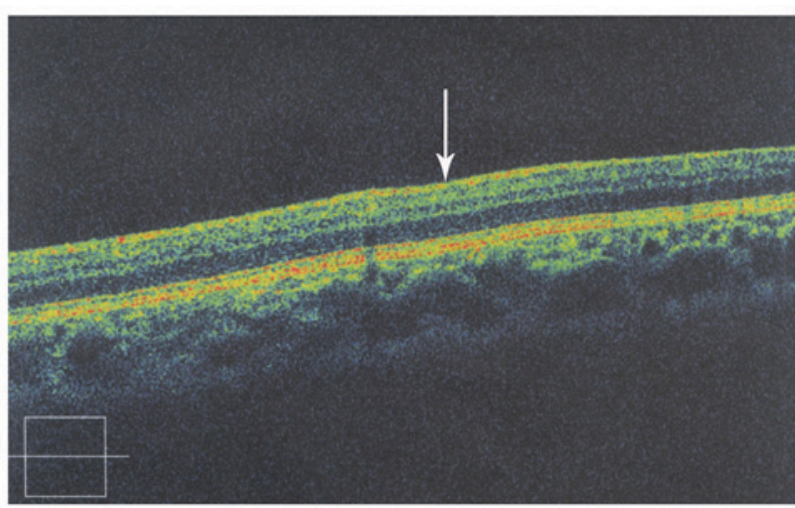

Figure 6. Representative optical coherence tomography examination in a patient with OA1. The foveal structure was not evident in any of the OA1 patients. White arrow indicates the absence of the foveal depression. OA1, ocular albinism type 1.

is the only identified pathogenic gene that may lead to the development of OA1 and, as it has few axons, this decreases the cost of genetic testing.

Furthermore, the positive screening rate of the GPR143 gene is relatively high. All of the above contribute to the relative ease of genetic testing for this gene and disease. The results from a genetic test may be used to further diagnose patients with existing clinical criteria and also aid in prenatal diagnosis.

The present study identified different degrees of hypopigmentation in the iris and retina, which was different from the sclera. Iris and fundus differences observed between the pigmented and white regions may be due to ethnic differences $(20,21)$. Therefore, according to the diagnostic criteria for OA1 in China, the use of clinical features based solely on the conditions of the sclera may lead to a misdiagnosis or missed diagnosis.

In conclusion, the findings of the present study expand the genetic mutation spectrum for the GPR143 gene and provide additional evidence for the clinical diagnosis, differential diagnosis and genetic counseling for OA1. Further investigation of the functional properties of the GPR143 protein, particularly by generating animal models, will provide information regarding how genetic mutations alter the molecular 
interactions involved in the generation of motor nystagmus. The analysis of these interactions may help to elucidate the underlying mechanisms of the disease.

\section{Acknowledgments}

The authors would like to thank the patients and family members for their participation. The present study was supported in part by the National Natural Science Foundation of China (grant nos. 81170847 and 81270972).

\section{References}

1. Schnur RE, Gao M, Wick PA, Keller M, Benke PJ, Edwards MJ, Grix AW, Hockey A, Jung JH, Kidd KK, et al: OA1 mutations and deletions in X-linked ocular albinism. Am J Hum Genet 62 : 800-809, 1998

2. Rosenberg T and Schwartz M: X-linked ocular albinism: Prevalence and mutations-a national study. Eur J Hum Genet 6: 570-577, 1998.

3. O'Donnell FE Jr, King RA, Green WR and Witkop CJ Jr: Autosomal recessively inherited ocular albinism. A new form of ocular albinism affecting females as severely as males. Arch Ophthalmol 96: 1621-1625, 1978.

4. Cortin P, Tremblay M and Lemagne JM: X-linked ocular albinism: Relative value of skin biopsy, iris transillumination and funduscopy in identifying affected males and carriers. Can J Ophthalmol 16: 121-123, 1981.

5. Lam BL, Fingert JH, Shutt BC, Singleton EM, Merin LM, Brown HH, Sheffield VC and Stone EM: Clinical and molecular characterization of a family affected with X-linked ocular albinism (OA1). Ophthalmic Genet 18: 175-184, 1997.

6. Sallmann GB, Bray PJ, Rogers S, Quince A, Cotton RG and Carden SM: Scanning the ocular albinism 1 (OA1) gene for polymorphisms in congenital nystagmus by DHPLC. Ophthalmic Genet 27: 43-49, 2006

7. Schiaffino MV, d'Addio M, Alloni A, Baschirotto C, Valetti C, Cortese K, Puri C, Bassi MT, Colla C, De Luca M, et al: Ocular albinism: Evidence for a defect in an intracellular signal transduction system. Nat Genet 23: 108-112, 1999.

8. Samaraweera P, Donatien PD, Qazi S, Kobayashi T, Hearing VJ, Panthier JJ and Orlow SJ: Identification and characterization of a melanocyte-specific novel $65-\mathrm{kDa}$ peripheral membrane protein. Eur J Biochem 266: 924-934, 1999.

9. d'Addio M, Pizzigoni A, Bassi MT, Baschirotto C, Valetti C, Incerti B, Clementi M, De Luca M, Ballabio A and Schiaffino MV: Defective intracellular transport and processing of OA1 is a major cause of ocular albinism type 1. Hum Mol Genet 9: 3011-3018, 2000.
10. Yan J and Dennin RH: High homologous nucleotide to GBV-C was amplified from DNA of MT2 and HeLa cells and PBMC of human and chimpanzee. Acta Pharmacol Sin 22: 320-326, 2001.

11. Zhang Q and Minoda K: Detection of congenital color vision defects using heteroduplex-SSCP analysis. Jpn J Ophthalmol 40: 79-85, 1996.

12. Stone EM: Leber congenital amaurosis-a model for efficient genetic testing of heterogeneous disorders: LXIV edward jackson memorial lecture. Am J Ophthalmol 144: 791-811, 2007.

13. Sale MM, Craig JE, Charlesworth JC, FitzGerald LM, Hanson IM, Dickinson JL, Matthews SJ, Heyningen Vv, Fingert JH and Mackey DA: Broad phenotypic variability in a single pedigree with a novel 1410 delC mutation in the PST domain of the PAX6 gene. Hum Mutat 20: 322, 2002.

14. Giordano F, Bonetti C, Surace EM, Marigo V and Raposo G: The ocular albinism type 1 (OA1) G-protein-coupled receptor functions with MART-1 at early stages of melanogenesis to control melanosome identity and composition. Hum Mol Genet 18: 4530-4545, 2009.

15. Bassi MT, Bergen AA, Bitoun P, Charles SJ, Clementi M, Gosselin R, Hurst J, Lewis RA, Lorenz B, Meitinger T, et al: Diverse prevalence of large deletions within the OA1 gene in ocular albinism type 1 patients from Europe and North America. Hum Genet 108: 51-54, 2001.

16. Schiaffino MV, Bassi MT, Galli L, Renieri A, Bruttini M, De Nigris F, Bergen AA, Charles SJ, Yates JR, Meindl A, et al: Analysis of the OA1 gene reveals mutations in only one-third of patients with X-linked ocular albinism. Hum Mol Genet 4: 2319-2325, 1995.

17. Rudolph G, Meindl A, Bechmann M, Schworm HD, Achatz H, Boergen KP, Kampik A, Berninger T and Meitinger T: X-linked ocular albinism (Nettleship-Falls): A novel 29-bp deletion in exon 1. Carrier detection by ophthalmic examination and DNA analysis. Graefes Arch Clin Exp Ophthalmol 239: 167-172, 2001.

18. Sone $\mathrm{M}$ and Orlow SJ: The ocular albinism type 1 gene product, OA1, spans intracellular membranes 7 times. Exp Eye Res 85: 806-816, 2007.

19. Camand O, Boutboul S, Arbogast L, Roche O, Sternberg C, Sutherland J, Levin A, Héon E, Menasche M, Dufier J and Abitbol M: Mutational analysis of the OA1 gene in ocular albinism. Ophthalmic Genet 24: 167-173, 2003.

20. O'Donnell FE Jr, Green WR, Fleischman JA and Hambrick GW: X-linked ocular albinism in Blacks. Ocular albinism cum pigmento. Arch Ophthalmol 96: 1189-1192, 1978.

21. Shiono T, Tsunoda M, Chida Y, Nakazawa M and Tamai M: X linked ocular albinism in Japanese patients. Br J Ophthalmol 79: 139-143, 1995. 